\title{
A Lagrangian approach to Chance Constrained Routing with Local Broadcast
}

\author{
Matteo Cacciola \\ Dipartimento di Informatica, Università di Pisa \\ Largo B.Pontecorvo 3, 56127 Pisa, Italia, m. cacciola1 estudenti.unipi.it \\ Antonio Frangioni \\ Dipartimento di Informatica, Università di Pisa \\ Largo B.Pontecorvo 3, 56127 Pisa, Italia, frangioddi.unipi.it \\ Laura Galli \\ Dipartimento di Informatica, Università di Pisa \\ Largo B.Pontecorvo 3, 56127 Pisa, Italia, laura.galli@unipi.it \\ Giovanni Stea \\ Dipartimento di Ingegneria dell'Informazione, Università di Pisa \\ Largo Lucio Lazzarino 1, 56122, Italia, giovanni. stea@unipi.it
}

January 24, 2020

LICENSE: Creative Commons: Attribution - Noncommercial - No Derivative Works

ADDRESS: Largo B. Pontecorvo 3, 56127 Pisa, Italy. $\quad$ TEL: +39 $0502212700 \quad$ FAX: +39 0502212726 



\title{
A Lagrangian approach to Chance Constrained Routing with Local Broadcast
}

\author{
Matteo Cacciola \\ Dipartimento di Informatica, Università di Pisa \\ Largo B.Pontecorvo 3, 56127 Pisa, Italia, m. cacciola1@studenti . unipi .it \\ Antonio Frangioni \\ Dipartimento di Informatica, Università di Pisa \\ Largo B.Pontecorvo 3, 56127 Pisa, Italia, frangioddi . unipi.it \\ Laura Galli \\ Dipartimento di Informatica, Università di Pisa \\ Largo B.Pontecorvo 3, 56127 Pisa, Italia, laura.galli@unipi.it \\ Giovanni Stea \\ Dipartimento di Ingegneria dell'Informazione, Università di Pisa \\ Largo Lucio Lazzarino 1, 56122, Italia, giovanni.stea@unipi.it
}

January 24, 2020

\begin{abstract}
Mobile cellular networks play a pivotal role in emerging Internet of Things (IoT) applications, such as vehicular collision alerts, malfunctioning alerts in Industry-4.0 manufacturing plants, periodic distribution of coordination information for swarming robots or platooning vehicles, etc. All these applications are characterized by the need of routing messages within a given local area (geographic proximity) with constraints about both timeliness and reliability (i.e., probability of reception). This paper presents a Non-Convex Mixed-Integer Nonlinear Programming model for a routing problem with probabilistic constraints on a wireless network. We propose an exact approach consisting of a branch-and-bound framework based on a novel Lagrangian decomposition to derive lower bounds. Preliminary experimental results indicate that the proposed algorithm is competitive with state-ofthe-art general-purpose solvers, and can provide better solutions than existing highly tailored ad-hoc heuristics to this problem.
\end{abstract}

Keywords: Internet of Things, Routing, Broadcast, Chance-Constrained Optimizations, Mixed-Integer Nonlinear Programs, Lagrangian relaxation, bundle methods, Branch-and-Bound

\section{Introduction}

Long Term Evolution Advanced (LTE-A) technology for cellular networks is the new forefront in the context of transmission networks for location-based broadcast services, such as advertising, smart-city applications, and Internet-of-Things (IoT) deployments. Yet, some new IoT services, such as vehicular collision alerts and augmentedreality live games, require low latency and high reliability, as well as the possibility to target an area defined by the application itself rather than the cell coverage. While traditional LTE-A tools can support these services, they do so at a rather large cost in terms of energy. In fact, on one hand, LTE's built-in Multicast/Broadcast mechanism was originally devised for broadcasting multimedia, and therefore unsuitable to this task because it is static: the message transmission format, the target area and the period of broadcast transmissions must all be selected statically. On the other hand, having the base station (antenna), called eNodeB (eNB) in the LTE terminology, relay messages 


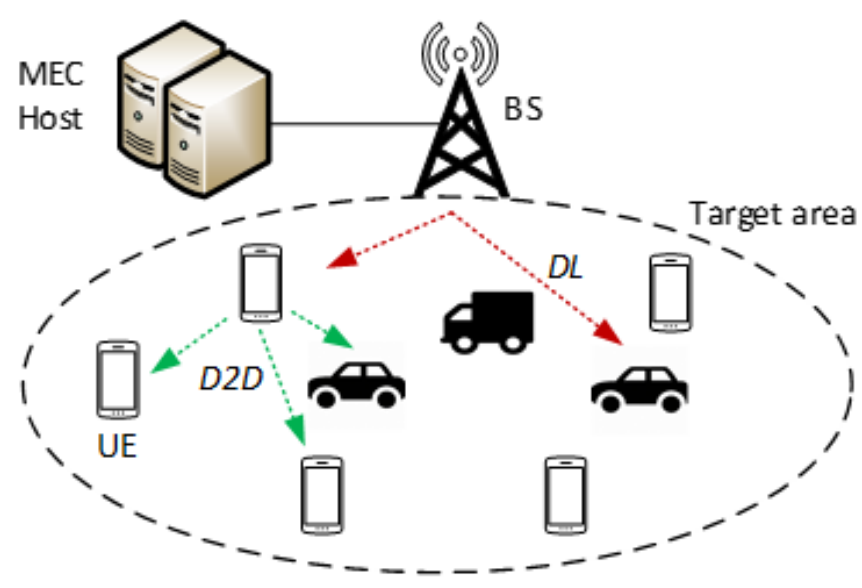

Figure 1: System model

to all the User Equipment (UEs) in a target area using unicast downlink (DL) transmissions (one per targeted UE) would require too many DL resources, hence too much energy. For this reason, recently, a new communication framework as been proposed. We consider a network of mobiles (UEs) which are under the control of a single eNB, as shown in Figure 1.

The eNB can send them information using DL (i.e., vertical) transmissions. Information can also travel through D2D links (i.e. horizontal broadcast transmissions originated at UEs). Vertical links are reliable but costly, and should be avoided if possible. By contrast, horizontal transmissions are free (from the eNB viewpoint), but not reliable: there is no ARQ mechanism involved, and it is impractical to try and ascertain which UEs, in the neighbourhood of the transmitter, have successfully decoded a message. However, UEs can act as multi-hop relays: horizontal transmissions are scheduled by the eNB, which issues grants to the UEs that may transmit. This allows to model with a reasonable accuracy the probability that a certain horizontal transmission is successful, given the position of the UEs, the transmission power of the transmitter, and the modulation and coding scheme adopted for transmission. This yields a new Chance-Constrained Unicast-Multicast Routing Problem (CCUMRP): select which vertical and horizontal multi-hop transmissions to choose in order to guarantee that all UEs receive the information with a certain level of reliability within a certain time limit, at minimum energy cost. We propose a Non-Convex Mixed-Integer Nonlinear Programming (MINLP) model for CCUMRP, together with an ad-hoc Lagrangian decomposition approach to compute lower bounds that separates the variable of the problem in a somewhat unusual fashion. We use the latter as the basis of a Branch-and-Bound (B\&B) approach that we computationally compare both with state-of-the-art, general-purpose exact solvers and with highly tailored ad-hoc heuristics for the problem.

\section{System Model}

We model the system as a graph $G=(N, A)$, where $N=\{0\} \cup N^{\prime}$ (0 being the eNB and $N^{\prime}$ representing the UEs) with $n=\left|N^{\prime}\right|$, while the arc set $A=A^{\prime} \cup A^{\prime \prime}$ consists of two types of arcs:

- vertical arcs $A^{\prime}$ of the form $(0, i)$ for all $i \in N^{\prime}$, representing a DL transmission between the eNB the UE $i$ having probability 1 to be decoded successfully at $i$ but high energy cost;

- horizontal arcs $A^{\prime \prime}$ of the form $(i, j)$ for $i \neq j \in N^{\prime}$, representing a D2D transmission from $i$ to $j$ having probability $0<P_{i j}<1$ to be decoded successfully at $j$, but low (energy) cost.

In the initial stage of the process the eNB transmits the message to a subset of UEs via DL (i.e., vertical transmission), while in the following stages only horizontal transmissions are allowed. A node $i \in N^{\prime}$ can only issue an horizontal transmission at a given stage if granted permission from the eNB. At most $M$ grants can be assigned in each stage, to ensure that the ensuing transmissions are not mutually interfering. The problem is therefore to transmit the message to the entire floorplan with the following constraints:

1. To ensure timeliness of reception of the message to all UEs, the broadcast process must be over in $k$ stages, 
with $k$ known a priori. Because the first round is clearly "special" (vertical transmissions from eNB to UEs), it is useful to define the set $K^{\prime}=\{2, \ldots, k\}$ of "normal" stages (horizontal transimissions between UEs).

2. To ensure reliability of reception, at the end of the broadcast process, each UE must possess the message with at least a given probability $\alpha$.

The main objective is to reduce the number of vertical transmissions. However, besides them, we also need to decide which UEs should transmit when (i.e., in which stage), so we must define the schedule of the grants that the eNB has to issue in order to compose the broadcast schedule. A secondary objective is to issue the least possible numbers of grants. In our model it is actually easy to generalize this by considering node-and-stage weighted grants costs $\beta_{i}^{h}\left(i \in N^{\prime}, h \in K^{\prime}\right)$, e.g., depending on the type of node $i$ and/or its remaining battery power.

\section{Mathematical Model}

We define the following set of variables:

- binary $x_{i}$ for $i \in N^{\prime}$ indicating whether node $i$ is selected in the initial set of UEs that are reached by the vertical transmission from eNB at stage 1;

- continuous $p_{i}^{h} \in[0,1]$ for $i \in N^{\prime}$ and $h \in K$ indicating the total probability that node $i$ has been reached at all stages up to $h$;

- binary $g_{i}^{h}$ for $i \in N^{\prime}$ and $h \in K^{\prime}$ indicating whether node $i$ is selected to receive a grant for broadcasting at stage $h$.

The MINLP model of CCUMRP is as follows:

$$
\begin{aligned}
& \min \sum_{i \in N^{\prime}} x_{i}+\sum_{h \in K^{\prime}} \sum_{i \in N^{\prime}} \beta_{i}^{h} g_{i}^{h} \\
& p_{i}^{1}=x_{i} \quad i \in N^{\prime} \\
& p_{i}^{k} \geq \alpha \quad i \in N^{\prime} \\
& 1-p_{i}^{h} \geq\left(1-p_{i}^{h-1}\right) \prod_{(j, i) \in A^{\prime \prime}}\left(1-g_{j}^{h} p_{j}^{h-1} P_{j i}\right) \quad i \in N^{\prime}, h \in K^{\prime} \\
& \sum_{i \in N^{\prime}} g_{i}^{h} \leq M \quad h \in K^{\prime} \\
& x_{i} \in\{0,1\} \\
& i \in N^{\prime} \\
& 0 \leq p_{i}^{h} \leq 1 \\
& g_{i}^{h} \in\{0,1\}
\end{aligned}
$$

The objective function (1) minimizes the number of initial vertical transmissions used in the first stage $(h=1)$ and the cost of grants issued during the subsequent stages $\left(h \in K^{\prime}\right)$; it is therefore intended that $\beta_{i}^{h} \ll 1$. Constraints (2) ensure that all initially targeted nodes are certainly reached. Constraints (3) impose that each UE node $i \in N^{\prime}$ is ultimately (at stage $k$ ) reached with probability at least $\alpha$; clearly, it would be trivial to generalize the model by allowing node-specific thresholds $\alpha_{i}$. Constraints (5) bound the total number of grants available at each stage (again, it would be trivial to let $M$ depend on $h$ ). Finally, the constrains characterizing the model are the nonlinear nonconvex (4) ones, expressing the probability that node $i$ at stage $h$ has not yet received the message.

Clearly, the problem would be almost trivial were it not for (4); therefore, it is those we will concentrate upon. Taking logarithms and noting that $g_{j}^{h}=0 \Longrightarrow \log \left(1-g_{j}^{h} p_{j}^{h-1} P_{j i}\right)=0$ they can be reduced to

$$
\log \left(1-p_{i}^{h}\right) \geq \log \left(1-p_{i}^{h-1}\right)+\sum_{(j, i) \in A^{\prime \prime}} g_{j}^{h} \log \left(1-p_{j}^{h-1} P_{j i}\right)
$$

which is at least linear with respect to variables $g_{j}^{h}$. However, the logarithm is ill-defined when $p_{i}^{h}=1$, which certainly happens at least whenever $x_{i}=1$. We therefore consider a restriction of the problem by selecting a constant $\bar{p}<1$ "arbitrarily close to 1 ", replacing (2) and (7), respectively, with

$$
\begin{array}{lr}
p_{i}^{1}=x_{i} \bar{p} & i \in N^{\prime} \\
0 \leq p_{i}^{h} \leq \bar{p} & i \in N^{\prime}, h \in K^{\prime}
\end{array}
$$


Clearly, each feasible solution of the new model is feasible for the original one as well, and by choosing $\bar{p}$ appropriately the practical difference between the two is poised to be minimal. Finally, let us mention for future reference that for the second stage (i.e., $h=2$ ) constraints (9) can be written in the form

$$
\log \left(1-p_{i}^{2}\right) \geq \log (1-\bar{p}) x_{i}+\sum_{(j, i) \in A^{\prime \prime}} g_{j}^{2} \log \left(1-P_{j i}\right) x_{j} \quad i \in N^{\prime},
$$

whose useful property is that the right-hand side does not contain any continuous variable (the $p_{i}^{1}$ having been substituted with the $x_{i}$ ). Therefore, (4) can be repalced by (9) for $h \in K^{\prime} \backslash\{2\}$ and by (12) for $h=2$. Nor that this, by itself, makes the constraints significantly easier to deal with. However, it allows us to propose a decomposition approaches to compute globally valid lower bounds.

\section{Lagrangian Decomposition}

We now discuss a Lagrangian decomposition of the MINLP formulation

$$
\begin{aligned}
& \min \sum_{i \in N^{\prime}} x_{i}+\sum_{h \in K^{\prime}} \sum_{i \in N^{\prime}} \beta_{i}^{h} g_{i}^{h} \\
& \quad(10),(3),(12),(9),(5), \text { (6) , (11), (8) }
\end{aligned}
$$

The idea is to relax constraints (12) and (9) with Lagrangian multipliers $\lambda_{i}^{h} \geq 0$ for $i \in N^{\prime}$ and $h \in K^{\prime}$. In so doing, the problem is decomposed into $k$ separate sub-problem; this is clearly due to the fact that (12)/(9) are the only constraints that link the variables of one stage to these of the following one. One may expect that each sub-problem has the variables corresponding to one specific level $h \in K$, but in fact the decomposition is somewhat different, and perhaps somewhat unusual. Indeed, each sub-problem actually has variables "of one kind" for one stage $h$ and variables "of another kind" for the subsequent stage $h+1$ (if any). This is due to the terms $g_{j}^{h} \log \left(1-p_{j}^{h-1} P_{j i}\right)$ in (9) (and, similarly, $g_{j}^{2} \log \left(1-P_{j i}\right) x_{j}$ in (12)) that link together variables $g_{j}^{h}$ with variables $p_{j}^{h-1}\left(x_{j}\right)$. We will now describe the sub-problems. Due to the special nature of the first stage, the corresponding sub-problem clearly has a particular structure. Not having a subsequent stage, the sub-problem corresponding to the last stage also has a peculiar form. All the sub-problems corresponding to intermediate stages rather share the same structure.

\subsection{Sub-problem $h=1$}

The first sub-problem contains the $x_{i}$ variables (that substitute for the probability variables $p_{i}^{1}$ ) of the first stage and the grant variables $g_{i}^{2}$ of the second stage, reading

$$
\begin{aligned}
& \min \sum_{i \in N^{\prime}}\left[x_{i}+\beta_{i}^{2} g_{i}^{2}+\lambda_{i}^{2}\left(\log (1-\bar{p}) x_{i}+\sum_{(j, i) \in A^{\prime \prime}} g_{j}^{2} \log \left(1-P_{j i}\right) x_{j}\right)\right] \\
& \sum_{i \in N^{\prime}} g_{i}^{2} \leq M \\
& x_{i}, g_{i}^{2} \in\{0,1\} \quad i \in N^{\prime}
\end{aligned}
$$

Collecting like terms of (13), and observing that there is no point in setting $g_{i}^{2}=1$ if $x_{i}=0$, yields:

$$
\begin{aligned}
& \min \sum_{i \in N^{\prime}}\left[\left(1+\lambda_{i}^{2} \log (1-\bar{p})\right) x_{i}+\left(\beta_{i}^{2}+\sum_{(i, j) \in A^{\prime \prime}} \lambda_{j}^{2} \log \left(1-P_{i j}\right)\right) g_{i}^{2}\right] \\
& \sum_{i \in N^{\prime}} g_{i}^{2} \leq M \\
& g_{i}^{2} \leq x_{i} \\
& x_{i}, g_{i}^{2} \in\{0,1\} \\
& \begin{array}{l}
i \in N^{\prime} \\
i \in N^{\prime}
\end{array}
\end{aligned}
$$

Since all nonlinear operations are applied to constants, the problem is linear. Furthermore, the special structure of the constraints ensures that, despite the variables being integer-valued, it can easily be solved in $\mathscr{O}(n \log n)$. 


\subsection{Sub-problems $2<h<k$}

Each of these sub-problems contains grant variables $g_{i}^{h+1}$ of stage $h+1$ and probability variables $p_{i}^{h}$ of stage $h$, reading

$$
\begin{aligned}
& \min \sum_{i \in N^{\prime}}\left[\left(\lambda_{i}^{h+1}-\lambda_{i}^{h}\right) \log \left(1-p_{i}^{h}\right)+\right. \\
& \left.\left(\beta_{i}^{h+1}+\sum_{(i, j) \in A^{\prime \prime}} \lambda_{j}^{h+1} \log \left(1-p_{i}^{h} P_{i j}\right)\right) g_{i}^{h+1}\right] \\
& 0 \leq p_{i}^{h} \leq \bar{p} \\
& i \in N^{\prime} \\
& \sum_{i \in N^{\prime}} g_{i}^{h+1} \leq M \\
& g_{i}^{h+1} \in\{0,1\}
\end{aligned}
$$

Clearly, in this problem each variable $p_{i}^{h}$ only interacts with the others via the single term in which it is multiplied by the corresponding $g_{i}^{h+1}$. The term is highly nonlinear, but still one can consider the corresponding function

$$
f_{i}^{h}(p, g)=\left(\lambda_{i}^{h+1}-\lambda_{i}^{h}\right) \log (1-p)+\left(\beta_{i}^{h+1}+\sum_{(i, j) \in A^{\prime \prime}} \lambda_{j}^{h+1} \log \left(1-p P_{i j}\right)\right) g
$$

By computing the two costants $p_{i}^{h, g}=\operatorname{argmin}\left\{f_{i}^{h}(p, g): 0 \leq p \leq \bar{p}\right\}$ for $g \in\{0,1\}$, the sub-problem can be rewritten as

$$
\min \left\{\sum_{i \in N^{\prime}} f_{i}^{h}\left(p_{i}^{h, 1}, 1\right) g_{i}^{h+1}+f_{i}^{h}\left(p_{i}^{h, 0}, 0\right)\left(1-g_{i}^{h+1}\right):(15),(16)\right\}
$$

and, therefore, again easily solved in $\mathscr{O}(n \log n)$. The crux of the subproblem therefore lies in the computation of $p_{i}^{h, 1}$ and $p_{i}^{h, 0}$. Computing the latter is trivial, as it reduces to minimizing on $p \in[0, \bar{p}]$ the monotone function $\left(\lambda_{i}^{h+1}-\lambda_{i}^{h}\right) \log (1-p)$; the optimum necessarily lies in one of the two extremes. Finding $p_{i}^{h, 1}$, instead, requires to tackle a more complex one-dimensional minimization problem of the form

$$
\min \left\{f(p)=c \log (1-p)+\sum_{i \in N^{\prime}} a_{i} \log \left(1-p b_{i}\right): 0 \leq p \leq \bar{p}\right\}
$$

whose solution will be discussed in $\S 5.1$.

\subsection{Sub-problem $k$}

Finally, the remaining Lagrangian term for $k$ is

$$
\min \left\{\sum_{i \in N^{\prime}}-\lambda_{i}^{k} \log \left(1-p_{i}^{k}\right): \alpha \leq p_{i}^{k} \leq \bar{p}\right\}
$$

that is separable over $i$; being the objective convex $\left(\lambda_{i}^{k} \geq 0\right)$, the optimum is in the left endpoint $p_{i}^{k}=\alpha$.

\section{Algorithmic approaches}

We now discuss all the relevant detail of the B\&B approach to CCUMRP that we developed using the proposed model and decomposition approach.

\subsection{Mono-dimensional problem}

The crucial step is clearly the ability to efficiently solve the one-dimensional problem (17). Note that $a_{i}=\lambda_{i}^{h+1} \geq 0$, $0 \leq P_{j i}=b_{i}<1$, while the coefficient $c=\lambda_{i}^{h+1}-\lambda_{i}^{h}$ is unrestricted in sign. Yet, if $c \geq 0$ then the problem is trivial: $f(p)$ is a decreasing function with $\lim _{p \rightarrow 1^{-}} f(p)=-\infty$, so the minimum is attained at $\bar{p}$. We will therefore concentrate on the case where $c<0$ instead, for which we will prove that there is at most one critical point $p_{0} \in[0, \bar{p}]$; moreover, the minimum is either attained at 0 or $p_{0}$. Indeed, $f(p)$ is the sum of the increasing function $c \log (1-p)(c<0)$ with vertical asymptote at $p=1$, and $n$ decreasing functions $a_{i} \log \left(1-p b_{i}\right)\left(a_{i} \geq 0\right)$ with vertical asymptotes at $p=1 / b_{i}>1$ (since $b_{i}<1$ ). Hence, clearly as $p \rightarrow 1$ the increasing function has to dominate: $\lim _{p \rightarrow 1^{-}} f(p)=+\infty$, and $f(p)$ has to be strictly increasing "close to" $\bar{p}$. As $p$ approaches 0 , instead, the behaviour depends on the $a_{i}$ values. In particular, we prove the following two cases: either the function is decreasing in 0 and becomes increasing "closer to" $\bar{p}$, which implies that the minimum is attained in the interior, or the function is increasing in 0 and remains so in the whole interval, which implies that the minimum is attained at 0 .

Lemma 1 If $c<0,0 \leq b_{i} \leq 1$ and $a_{i} \geq 0$ then there exists at most one critical point $p_{0} \in[0,1)$ such that $f^{\prime}\left(p_{0}\right)=0$, and $f(p)$ is strictly increasing in $p_{0}<p \leq 1$. 
Proof. Consider $f^{\prime}(p)=-\frac{c}{1-p}-\sum_{i \in N^{\prime}} \frac{a_{i} b_{i}}{1-p b_{i}}$, we have

$$
f^{\prime}(p) \geq 0 \quad \Longleftrightarrow \quad-\sum_{i \in N^{\prime}} \frac{(1-p) a_{i} b_{i}}{\left(1-p b_{i}\right) c}=h(p) \leq 1
$$

It is now immediate to see that

$$
h^{\prime}(p)=-\sum_{i \in N^{\prime}} \frac{a_{i} b_{i}}{c} \frac{b_{i}-1}{\left(1-p b_{i}\right)^{2}} \leq 0
$$

for all $p \in[0,1]$. This means that there can be at most one point $p_{0} \in[0,1)$ such that $f^{\prime}\left(p_{0}\right)=0$, and therefore $f(p)$ is strictly increasing in $\left(p_{0}, 1\right)$.

We now analyse convexity of $f$, showing that if the function is non-convex then there is exactly one point $\hat{p}$ in which the second derivative changes its sign, and that the function is convex in $[\hat{p}, 1]$.

Lemma 2 If $c<0,0 \leq b_{i} \leq 1$ and $a_{i} \geq 0$, then there exists at most one point $\hat{p} \in[0,1)$ with $f^{\prime \prime}(\hat{p})=0$, and $f(p)$ is convex in $\hat{p} \leq p \leq 1$.

Proof. Along the same lines, for $f^{\prime \prime}(p)=-\frac{c}{(1-p)^{2}}-\sum_{i \in N^{\prime}} \frac{a_{i} b_{i}^{2}}{\left(1-p b_{i}\right)^{2}}$ we have

$$
f^{\prime \prime}(p) \geq 0 \quad \Longleftrightarrow \quad-\sum_{i \in N^{\prime}} \frac{(1-p)^{2} a_{i} b_{i}^{2}}{\left(1-p b_{i}\right)^{2} c}=h(p) \leq 1
$$

which similarly yields

$$
h^{\prime}(p)=-\sum_{i \in N^{\prime}} \frac{a_{i} b_{i}^{2}}{c} 2(1-p)\left(1-p b_{i}\right) \frac{b_{i}-1}{\left(1-p b_{i}\right)^{4}}<0
$$

for all $p \in[0,1]$. Again, this implies that if $f^{\prime \prime}(\hat{p})=0$ for some $\hat{p} \in[0,1)$, then $f^{\prime \prime}(p) \geq 0$ (i.e., $f$ is convex) for all $\hat{p} \leq p \leq 1$

To recap, the following three cases can happen:

1. $f(p)$ is increasing in $[0, \bar{p}]$, hence the minimum is 0 ;

2. $f(p)$ is decreasing in 0 but convex in $[0, \bar{p}]$, hence the minimum is in the interior of the interval;

3. $f(p)$ is decreasing in 0 and convex in $[\hat{p}, \bar{p}]$ for some $\hat{p}>0$, hence the minimum lies in the interval $[\hat{p}, \bar{p}]$; that are represented in Figures 2, 3, 4, respectively.

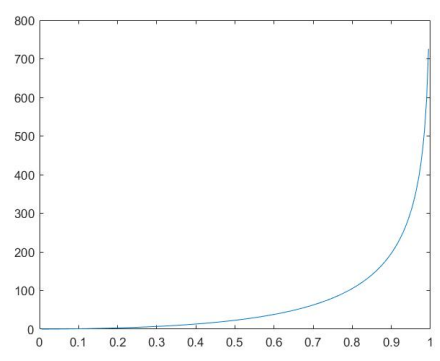

Figure 2: Increasing

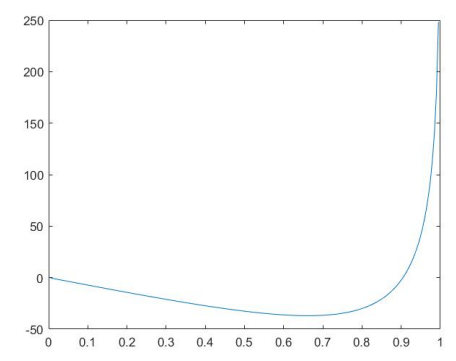

Figure 3: Convex

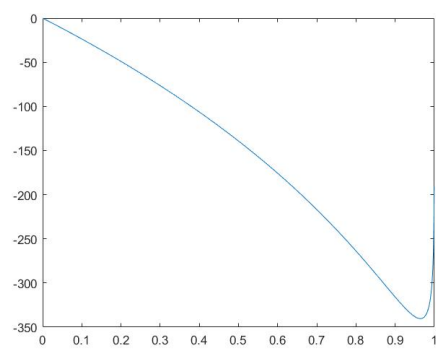

Figure 4: Concave, then convex

From an algorithmic viewpoint, such a function can be efficiently globally minimized using a simple globalization of Newton's method. We keep an interval $\left[p_{-}, p_{+}\right]$such that $f^{\prime}\left(p_{-}\right)<0$ and $f^{\prime}\left(p_{+}\right)>0$ (initialized as $[0, \bar{p}]$, unless if $f^{\prime}(0) \geq 0$ in which case we immediately terminate). If $f^{\prime \prime}\left(p_{-}\right)<0\left(f\right.$ is non convex at $\left.p_{-}\right)$we use a simple bisection rule to find a point $p_{-}<p^{\prime}<p_{+}$, we compute $f^{\prime}\left(p^{\prime}\right)$ and shrink the interval accordingly. Otherwise ( $f$ is convex at $p_{-}$) we compute Newton's step, and we accept it if it belongs to the interval and it shrinks it enough; otherwise we revert to the simple bisection rule. This is clearly convergent, and typically quadratically so in the tail. Note that in our function the minimum is often close to 1 , so instead of using a standard bisection we use the point $p^{\prime}=p_{-}+3 / 4\left(p_{+}-p_{-}\right)$, as this typically leads to faster initial convergence. 


\subsection{Lagrangian dual problem}

It is well-known that for each choice of $\lambda \in \mathbb{R}_{+}^{2 n}$, the solution of the corresponding Lagrangian relaxation provides a valid global lower bound on the optimal value of the original problem. To find the best possible Lagrangian relaxation, one then has to solve the Lagrangian Dual problem, i.e., maximize over all $\lambda \geq 0$ the Lagrangian function consisting of the $k$ terms described in $\S 4.1,4.2$ and 4.3. Even with the best possible choice $\lambda^{*}$ of the Lagrangian multipliers, there is no guarantee that the penalty terms in the objective function will lead to a feasible integer solution, i.e., one that satisfies the relaxed constraints (9) and (12); however, it is well-known that the Lagrangian Dual is equivalent to the convexified relaxation of the original problem [4]. The efficiency of the solution process obviously depend on the specific algorithm used to solve the Lagrangian Dual; in our case we use the freely available implementation of the (generailized) [3] proximal Bundle method already used with success in other applications (e.g., $[7,6])$ provided by the NDOSolver/FiOracle suite of C++ solvers for NonDifferentiable Optimization problems developed by the Department of Computer Science of the University of Pisa [10].

While we refer to [3] and [10] for details on Bundle methods, we briefly point out two features that are particularly relevant in our application:

- These methods sample the objective function in a sequence of points and store (a subset of) the first-order information (subgradients) obtained at each. In the Lagrangian setting, a subgradient corresponds to an optimal (integer) solution of the Lagrangian subproblem(s). These can be stored alongside the subgradients (the NDOSolver/FiOracle framework providing appropriate support). At the end (but, actually, at each iteration) they can therefore be used as starting points for heuristic approaches (cf. $\S 5.3$ ); even more significantly, they can be used to obtain the optimal solution of the convexified relaxation corresponding to the Lagrangian dual [4], which can again be useful for heuristic purposes (cf. again §5.3) and is crucial for branching decisions (cf. §5.4).

- These methods can also work in a disaggregate version exploiting any existing sum-structure of the objective function, as in our application. This typically results in a much improved convergence speed (e.g., [2,6]), at the cost of a more expensive master problem. Thus, whether or not the disaggregate version performs better than the aggregate one depends on the fine details of the application. In our case, the disaggregate version was most often faster, reducing by up to 6 times the number of iterations w.r.t. the aggregate one.

\subsection{Heuristics}

A fundamental component of any partial enumeration approach is the ability of producing good feasible solutions that can be used to prune nodes of the decision tree (and that ultimately provide the returned best solution). We can do this, potentially at each iteration, using both the integer, but (typically) not feasible, solution that we obtain by computing the Lagrangian function, and the continuous, but (quickly) "almost feasible", convexified solution that can be obtained as a by-product of solving the master problem in the Bundle method [4]. Actually, exploiting both synergistically has been shown to be useful in some applications [2, 5].

We will start discussing the use of the integer solution obtained from the computation of the Lagrangian function. We can clearly ignore the continuous variables $p_{j}^{h}$, since we can compute them from the $x_{i}$ and $g_{i}^{h}$ ones so that constraints (4) are satisfied. Moreover, the constraints (5) are naturally satisfied since they are a part of the Lagrangian subproblems. The only constraints that can be not satisfied are (obviously) the relaxed (3) ones; this means that there are not enough transmission to ensure that all users receive the message with probability at least $\alpha$. An obvious idea (reminiscent of that of $[2,5]$ in a completely different setting) is then to use the unfeasible solution as starting point and fix more variables to 1 to make it feasible. Given an integer solution, we define the score of integer variables as follows:

$$
\begin{aligned}
& S\left(x_{i}\right)=\sum_{j: p_{j}^{k}<\alpha} P_{i, j}^{2}\left(\alpha-p_{j}^{k}\right)^{3} \delta_{i} \\
& S\left(g_{i}^{h}\right)=\sum_{j: p_{j}^{k}<\alpha} P_{i, j}^{2}\left(\alpha-p_{j}^{k}\right)^{3} p_{i}^{h-1}
\end{aligned}
$$

Intuitively, the higher is the connection $\left(P_{i, j}\right)$ of a user with the other ones that did not receive the message, the higher is the score of the integer variables related to it. On the other hand, being connected with a users that have almost received the signals is less important that being connected with another one that is way far from receiving it, which is the rationale behind the addition of the term $\left(\alpha-p_{j}^{k}\right)$, i.e., the gap between the actual value of $p_{j}^{k}$ and the minimum that it have to take to be feasible. Since the $x_{i}$ variables have much larger cost than the $g_{i}^{h}$ ones $\left(\beta_{i}^{h} \ll 1\right)$, we reflect this by a proper scaling factor $\delta_{i}<1$ (if the costs $\beta_{i}^{h}$ are constant with the step we can use 
$\left.\delta_{i}=\beta_{i}\right)$. For the $g_{i}^{h}$ variables, another important factor is the probability that the user $i$ received the signal at step $h-1$, as it is transmitting from a user that has received the signal with low probability is unlikely to be efficient; this is the rationale behind the extra term $p_{j}^{h-1}$. The exponents of these factors could be changed to give them more or less impact in the final score. Having computed the score, the heuristic is a simple greedy one: until we reach a feasible solution, we activate the inactive variable with the best score that does not violate (5). This algorithm can be used starting from every integer solution that satisfies (5), such as the solution of the Lagrangian relaxation and the null solution.

Another tool that we can use to find an heuristic solution is the convexified solution obtained when we solve the Lagrangian dual. This is a fractional solution, that can be rounded in order to find an integer one. The rounding strategy we tried is basically to set to 1 the $m_{0} x_{i}$ variables having higher (fractional) value in the convexified solution, and, likewise, setting to 1 the $m_{h} g_{i}^{h}$ variables having higher value for each step $h \in K^{\prime}$. The choice of the parameters $m_{h}$ leads the heuristic solution in different directions: $m_{0}$ influence how many vertical communications there will be at step 1, hence a too small value can easily lead to an infeasible solution, but on the other hand a too large one will typically result in inefficient ones. Unevenly choosing $m_{h}$ can lead either to solutions that prefer to transmit the signal in the initial steps to make the future transmission more effective, or to solutions that wait the advanced steps, when the signal is more spread, to make a large number of transmissions.

Unfortunately, all the simple rounding strategies had issues to find feasible solutions. We were still able to construct feasible solutions by using the thusly constructed integer solution as the initial one in the previously discussed greedy heuristic. We tested several combination of the parameters in these heuristics, and found that those that are based on the convexified solution are typically not competitive with these that directly start from an integer solution. This (somewhat disappointing, in view of much better success obtained in $[2,5]$ ) result is probably due to the fact that the "quality" of the convexified solution is not very high: the solution is highly fractional, corresponding to the fact that the bound is not very tight, as discussed in $\S 6$.

\subsection{Branch-and-bound}

Since both upper and lower bounds obtained with the methods previously discussed are not very tight, we implemented an implicit enumeration (Branch-and-Bound) algorithm in order to obtain better gaps.

Most of the implementation choices in the B\&B are fairly straightforward. At each node we solve the Lagrangian Dual with a time limit of 1 second, as experience has shown that the dual solution obtained after that time was typically, if not optimal, good enough as to no warrant further effort. Note that since all values $\beta_{i}^{h}$ are fixed to 0.2 in our instances, we can round up the lower bound to the nearest multiple of 0.2. At the end of this process we run the heuristics based on all the integer solutions that are in the bundle.

The branching rule we used is the standard one of choosing a variable and fix it to 1 in one son and to 0 in the other. To decide the variable that will be fixed we look at the convexified solution and we choose the fractional $x$ variable that has the closest value to 0.5 ; if all the $x$ variables are already fixed, or take integer values in the convexified solution, we choose a $g$ variable with a similar rule. Branching first on the $x$ variables makes sense since they are those that are likely to have the most impact on the solution of the problem.

The visit strategy of the decision tree is the standard "best first": we pick the node that have the lowest lower bound. The use of best first was dictated by to the relatively poor quality of the lower bound, as this is the strategy that is known to usually improve it the most.

\section{Computational Results}

We tested the model on the realistic scenarios constructed with the help of the SimuLTE simulator developed at the Department of Information Engineering of the University of Pisa [11]. The tool allows to create many instances of the problem tuning the main parameters of interests; in our experiments we mainly concentrated on the number of UEs, on the radius (in meters) of the geographical region of interest, and on the required coverage probability $\alpha$.

We compared our Lagrangian-based B\&B with the state-of-the-art, general-purpose MINLP solver BARON [1] 18.11.12, as well as with a highly-tailored combinatorial heuristic available in SimuLTE and described in $[8,9]$. For BARON, we scaled the objective function by a factor of 5 so that all the coefficients are integer, allowing it to also exploit integrality to round up the lower bound. All codes have been compiled with $9++7.4 .0$ and ran single-threaded on a machine sporting a 16-core Intel Xeon5120 CPU@2.20GHz and 64Gb RAM, running Ubuntu 18.04. The results are reported in the following Tables, with two different time limits: 300 seconds and 
3000 seconds. The instances are characterized by the number of UEs ("\#”), the radius (" $r$ ") and the covering probability (" $\alpha$ "). For both exact methods we report the total running time ("time") if they terminated before the time limit, and "-" otherwise, plus the total number of B\&B nodes ("nodes"). We also report the inherent gap ("gap"), i.e., $(U B-L B) / \max \{1, L B\}$ (in percentage), where $U B$ and $L B$ are the best upper and lower bound on the optimal value produced by the corresponding algorithm at termination. To better represent the relative quality of the upper and lower bounds, we also separately report the primal gap ("pgap") $(U B-\underline{U B}) / \max \{1, \underline{U B}\}$ and the dual gap ("dgap") $(\overline{L B}-L B) / \max \{1, L B\}$ (in percentage), where $\underline{U B}$ and $\overline{L B}$ are, respectively, the best (lowest) known upper bound and best (highest) known lower bound on the optimal value of the instance. Note that since the largest and hardest instances were not solved within 3000 seconds, a 0 primal or dual gap does necessarily means that the corresponding $U B / L B$ are the optimal value, but only that they are the best ever found in our experiments.

The results in Table 1 clearly show how challenging CCUMRP is. Only 10-UEs instances can be all solved to optimality by our approach within the 5-minutes time limit; it is generally more efficient than BARON (which fails to solve two) except for very large $r$, where BARON closes at root node. Interestingly, the combinatorial heuristicwhich is the state-of-the-art for the problem up until this work-provides solutions that can be in excess of $50 \%$ off the optimum, although of course does so in orders-of-magnitude less time. When the size of the instances grows, BARON is basically unable to solve the problem except in a handful of cases, providing both lower and especially upper bounds that are of no practical value. Our approach cannot be exactly deemed to be very successful, with final gaps up to $40 \%$ with 25 users and even in excess of $200 \%$ with 50 users; however, it still produces the best solutions and lower bounds.

Moving to the time limit of 3000 seconds, depicted in Table 2, confirms that our approach at least scales much better than BARON; the much faster bound computation allows to enumerate more B\&B nodes, which ultimately results in much better upper and lower bounds. In particular, we are able to solve about half of the instances with 25 users to optimality, with the other half ending with "reasonable" gaps (at least, if compared with these of BARON, both lower and upper, and with the upper bounds provided by the combinatorial heuristic). All in all, our approach is only partly successful. In particular, the lower bound is not particularly tight, which limits the size of the instances that can be practically solved. However, it at least provides a way to assess the performances of the heuristics approaches which, due to the extremely tight time limits (a handful of milliseconds) imposed by the application, are probably the only practical way of approaching the problem. Hopefully, the information provided by our approach will allow to better identify the limits of the current heuristics, and develop better ones.

\section{Acknowledgement}

The authors gratefully acknowledge the partial financial support from the Italian Ministry of Education, University and Research (MIUR), under the project "Nonlinear and combinatorial aspects of complex networks" (grant PRIN 2015B5F27W).

\section{References}

[1] The BARON solver, https://minlp.com/baron.

[2] A. Borghetti, A. Frangioni, F. Lacalandra, and C.A. Nucci. Lagrangian Heuristics Based on Disaggregated Bundle Methods for Hydrothermal Unit Commitment. IEEE Transactions on Power Systems, 18(1):313-323, February 2003.

[3] A. Frangioni. Generalized Bundle Methods. SIAM Journal on Optimization, 13(1):117-156, 2002.

[4] A. Frangioni. About Lagrangian Methods in Integer Optimization. Annals of Operations Research, 139(1):163-193, 2005.

[5] A. Frangioni, C. Gentile, and F. Lacalandra. Solving Unit Commitment Problems with General Ramp Contraints. International Journal of Electrical Power and Energy Systems, 30:316-326, 2008.

[6] A. Frangioni and E. Gorgone. Generalized Bundle Methods for Sum-Functions with "Easy" Components: Applications to Multicommodity Network Design. Mathematical Programming, 145(1):133-161, 2014.

[7] A. Frangioni, A. Lodi, and G. Rinaldi. New approaches for optimizing over the semimetric polytope. Mathematical Programming, 104(2-3):375-388, 2005. 


\begin{tabular}{|c|c|c|c|c|c|c|c|c|c|c|c|c|c|}
\hline \multicolumn{3}{|c|}{ Instances } & \multicolumn{5}{|c|}{ BARON } & \multicolumn{5}{|c|}{$\mathrm{B} \& \mathrm{~B}$} & \multirow{2}{*}{$\frac{\mathrm{CH}}{\text { pgap }}$} \\
\hline \# & $r$ & & time & nodes & gap & pgap & dgap & time & nodes & gap & pgap & dgap & \\
\hline 10 & 100 & 0.92 & 4.86 & 1 & 0.00 & 0.00 & 0.00 & 0.59 & 20 & 0.00 & 0.00 & 0.00 & 0.00 \\
\hline 10 & 100 & 0.95 & 3.07 & 1 & 0.00 & 0.00 & 0.00 & 0.58 & 20 & 0.00 & 0.00 & 0.00 & 0.00 \\
\hline 10 & 100 & 0.96 & 3.43 & 1 & 0.00 & 0.00 & 0.00 & 0.67 & 20 & 0.00 & 0.00 & 0.00 & 0.00 \\
\hline 10 & 250 & 0.92 & 4.92 & 1 & 0.00 & 0.00 & 0.00 & 0.44 & 20 & 0.00 & 0.00 & 0.00 & 0.00 \\
\hline 10 & 250 & 0.95 & 75.39 & 1 & 0.00 & 0.00 & 0.00 & 0.73 & 20 & 0.00 & 0.00 & 0.00 & 0.00 \\
\hline 10 & 250 & 0.96 & 31.32 & 1 & 0.00 & 0.00 & 0.00 & 0.46 & 20 & 0.00 & 0.00 & 0.00 & 71.4 \\
\hline 10 & 500 & 0.92 & 0.67 & 84 & 0.00 & 0.00 & 0.00 & 193.8 & 12323 & 0.00 & 0.00 & 0.00 & 71.4 \\
\hline 10 & 500 & 0.95 & 4.45 & 52 & 0.00 & 0.00 & 0.00 & 44.00 & 2717 & 0.00 & 0.00 & 0.00 & 40.0 \\
\hline 10 & 500 & 0.96 & 383.4 & 1597 & 0.00 & 0.00 & 0.00 & 229.1 & 5130 & 0.00 & 0.00 & 0.00 & 46.7 \\
\hline 10 & 750 & 0.92 & 269.2 & 1778 & 0.00 & 0.00 & 0.00 & 153.42 & 2402 & 0.00 & 0.00 & 0.00 & 29.4 \\
\hline 10 & 750 & 0.95 & - & 715 & 4.00 & 0.00 & 4.00 & 208.5 & 6880 & 0.00 & 0.00 & 0.00 & 38.5 \\
\hline 10 & 750 & 0.96 & - & 717 & 13.0 & 0.00 & 13.0 & 29.87 & 1026 & 0.00 & 0.00 & 0.00 & 50.0 \\
\hline 10 & 1000 & 0.92 & 1.78 & 1 & 0.00 & 0.00 & 0.00 & 79.81 & 2913 & 0.00 & 0.00 & 0.00 & 26.9 \\
\hline 10 & 1000 & 0.95 & 1.42 & 1 & 0.00 & 0.00 & 0.00 & 210.0 & 13754 & 0.00 & 0.00 & 0.00 & 36.4 \\
\hline 10 & 1000 & 0.96 & 0.82 & 1 & 0.00 & 0.00 & 0.00 & 1.91 & 120 & 0.00 & 0.00 & 0.00 & 63.6 \\
\hline 25 & 100 & 0.92 & - & 1 & 3780 & 3050 & 120 & 121.6 & 164 & 0.00 & 0.00 & 0.00 & 0.00 \\
\hline 25 & 100 & 0.95 & - & 17 & 100 & 0.00 & 100 & 98.45 & 130 & 0.00 & 0.00 & 0.00 & 0.00 \\
\hline 25 & 100 & 0.96 & - & 18 & 80.0 & 0.00 & 80 & 57.56 & 84 & 0.00 & 0.00 & 0.00 & 0.00 \\
\hline 25 & 250 & 0.92 & - & 1 & 3780 & 3050 & 120 & 12.83 & 58 & 0.00 & 0.00 & 0.00 & 0.00 \\
\hline 25 & 250 & 0.95 & - & 1 & 3780 & 2600 & 140 & 11.30 & 58 & 0.00 & 0.00 & 0.00 & 0.00 \\
\hline 25 & 250 & 0.96 & - & 1 & 3780 & 2600 & 140 & 10.04 & 56 & 0.00 & 0.00 & 0.00 & 0.00 \\
\hline 25 & 500 & 0.92 & - & 1 & 3780 & 1354 & 260 & - & 1995 & 40.0 & 7.69 & 30.0 & 30.8 \\
\hline 25 & 500 & 0.95 & - & 1 & 3780 & 1354 & 260 & - & 1983 & 23.1 & 23.1 & 0.00 & 69.2 \\
\hline 25 & 500 & 0.96 & - & 1 & 3780 & 1250 & 260 & - & 1569 & 23.1 & 14.3 & 0.00 & 42.9 \\
\hline 25 & 750 & 0.92 & - & 2 & 1160 & 456 & 80 & - & 642 & 40.0 & 2.94 & 8.00 & 32.4 \\
\hline 25 & 750 & 0.95 & - & 5 & 1081 & 425 & 88 & - & 1263 & 23.3 & 2.78 & 0.00 & 22.2 \\
\hline 25 & 750 & 0.96 & - & 4 & 1081 & 425 & 88 & - & 1004 & 23.3 & 2.78 & 0.00 & 22.2 \\
\hline 25 & 1000 & 0.92 & - & 12 & 330 & 210 & 25 & - & 1332 & 14.6 & 3.28 & 0.00 & 36.2 \\
\hline 25 & 1000 & 0.95 & - & 10 & 311 & 205 & 26 & - & 1185 & 12.5 & 1.61 & 3.57 & 29.0 \\
\hline 25 & 1000 & 0.96 & - & 12 & 294 & 200 & 25 & - & 951 & 12.3 & 1.59 & 5.26 & 47.6 \\
\hline 50 & 100 & 0.92 & - & 1 & 6280 & 5133 & 40 & - & 283 & 100 & 0.00 & 20.0 & 0.00 \\
\hline 50 & 100 & 0.95 & - & 1 & 6280 & 5133 & 40 & - & 283 & 100 & 0.00 & 20.0 & 0.00 \\
\hline 50 & 100 & 0.96 & - & 1 & 6280 & 5133 & 40 & - & 283 & 100 & 0.00 & 20.0 & 0.00 \\
\hline 50 & 250 & 0.92 & - & 1 & 780 & 550 & 80 & - & 283 & 60.0 & 0.00 & 20.0 & 0.00 \\
\hline 50 & 250 & 0.95 & - & 1 & 6280 & 4386 & 80 & - & 283 & 80.0 & 0.00 & 20.0 & 0.00 \\
\hline 50 & 250 & 0.96 & - & 1 & 6280 & 4386 & 80 & - & 284 & 80.0 & 0.00 & 20.0 & 0.00 \\
\hline 50 & 500 & 0.92 & - & 1 & 6280 & 2143 & 140 & - & 283 & 180 & 0.00 & 40.0 & 21.4 \\
\hline 50 & 500 & 0.95 & - & 1 & 6280 & 1993 & 160 & - & 284 & 114 & 0.00 & 14.3 & 20.0 \\
\hline 50 & 500 & 0.96 & - & 1 & 6280 & 1993 & 160 & - & 283 & 87.5 & 0.00 & 0.00 & 20.0 \\
\hline 50 & 750 & 0.92 & - & 1 & 6280 & 913 & 200 & - & 292 & 230 & 6.45 & 0.00 & 3.20 \\
\hline 50 & 750 & 0.95 & - & 1 & 6280 & 772 & 260 & - & 291 & 192 & 5.56 & 0.00 & 22.2 \\
\hline 50 & 750 & 0.96 & - & 1 & 6280 & 749 & 260 & - & 290 & 185 & 0.00 & 0.00 & 18.9 \\
\hline 50 & 1000 & 0.92 & - & 1 & 6280 & 398 & 560 & - & 283 & 220 & 1.59 & 40.0 & 11.1 \\
\hline 50 & 1000 & 0.95 & - & 1 & 6280 & 376 & 600 & - & 280 & 156 & 4.55 & 11.1 & 28.8 \\
\hline 50 & 1000 & 0.96 & - & 1 & 6280 & 355 & 700 & - & 280 & 154 & 2.90 & 25.0 & 31.9 \\
\hline
\end{tabular}

Table 1: Computational results, time limit 300 seconds 


\begin{tabular}{|c|c|c|c|c|c|c|c|c|c|c|c|c|c|}
\hline \multicolumn{3}{|c|}{ Instances } & \multicolumn{5}{|c|}{ BARON } & \multicolumn{5}{|c|}{$\mathrm{B} \& \mathrm{~B}$} & $\mathrm{Cl}$ \\
\hline \# & $r$ & & time & nodes & gap & pgap & dgap & time & nodes & gap & pgap & dgap & pgap \\
\hline 10 & 100 & 0.92 & 5.13 & 1 & 0.00 & 0.00 & 0.00 & 0.59 & 20 & 0.00 & 0.00 & 0.00 & 0.00 \\
\hline 10 & 100 & 0.95 & 3.12 & 1 & 0.00 & 0.00 & 0.00 & 0.58 & 20 & 0.00 & 0.00 & 0.00 & 0.00 \\
\hline 10 & 100 & 0.96 & 3.48 & 1 & 0.00 & 0.00 & 0.00 & 0.67 & 20 & 0.00 & 0.00 & 0.00 & 0.00 \\
\hline 10 & 250 & 0.92 & 4.69 & 1 & 0.00 & 0.00 & 0.00 & 0.44 & 20 & 0.00 & 0.00 & 0.00 & 0.00 \\
\hline 10 & 250 & 0.95 & 75.22 & 1 & 0.00 & 0.00 & 0.00 & 0.73 & 20 & 0.00 & 0.00 & 0.00 & 0.00 \\
\hline 10 & 250 & 0.96 & 1.12 & 1 & 0.00 & 0.00 & 0.00 & 0.46 & 20 & 0.00 & 0.00 & 0.00 & 71.4 \\
\hline 10 & 500 & 0.92 & 2.39 & 84 & 0.00 & 0.00 & 0.00 & 193.7 & 12323 & 0.00 & 0.00 & 0.00 & 71.4 \\
\hline 10 & 500 & 0.95 & 46.08 & 52 & 0.00 & 0.00 & 0.00 & 44.0 & 2717 & 0.00 & 0.00 & 0.00 & 40.0 \\
\hline 10 & 500 & 0.96 & 383.4 & 1597 & 0.00 & 0.00 & 0.00 & 229.1 & 5130 & 0.00 & 0.00 & 0.00 & 46.7 \\
\hline 10 & 750 & 0.92 & 269.2 & 1778 & 0.00 & 0.00 & 0.00 & 153.42 & 2402 & 0.00 & 0.00 & 0.00 & 29.4 \\
\hline 10 & 750 & 0.95 & 439.0 & 911 & 0.00 & 0.00 & 0.00 & 208.5 & 6880 & 0.00 & 0.00 & 0.00 & 38.5 \\
\hline 10 & 750 & 0.96 & 1456 & 2605 & 0.00 & 0.00 & 0.00 & 29.9 & 1026 & 0.00 & 0.00 & 0.00 & 50.0 \\
\hline 10 & 1000 & 0.92 & 1.78 & 1 & 0.00 & 0.00 & 0.00 & 79.81 & 2913 & 0.00 & 0.00 & 0.00 & 26.9 \\
\hline 10 & 1000 & 0.95 & 1.66 & 1 & 0.00 & 0.00 & 0.00 & 210.0 & 13754 & 0.00 & 0.00 & 0.00 & 36.4 \\
\hline 10 & 1000 & 0.96 & 0.90 & 1 & 0.00 & 0.00 & 0.00 & 1.91 & 120 & 0.00 & 0.00 & 0.00 & 63.6 \\
\hline 25 & 100 & 0.92 & - & 71 & 100 & 0.00 & 100 & 121.6 & 164 & 0.00 & 0.00 & 0.00 & 0.00 \\
\hline 25 & 100 & 0.95 & - & 94 & 80.0 & 0.00 & 80.0 & 98.5 & 130 & 0.00 & 0.00 & 0.00 & 0.00 \\
\hline 25 & 100 & 0.96 & - & 109 & 80.0 & 0.00 & 80.0 & 57.6 & 84 & 0.00 & 0.00 & 0.00 & 0.00 \\
\hline 25 & 250 & 0.92 & - & 107 & 80.0 & 0.00 & 80.0 & 12.8 & 58 & 0.00 & 0.00 & 0.00 & 0.00 \\
\hline 25 & 250 & 0.95 & - & 21 & 100 & 0.00 & 100 & 11.3 & 58 & 0.00 & 0.00 & 0.00 & 0.00 \\
\hline 25 & 250 & 0.96 & - & 18 & 100 & 0.00 & 100 & 10.0 & 56 & 0.00 & 0.00 & 0.00 & 0.00 \\
\hline 25 & 500 & 0.92 & - & 29 & 3680 & 1354 & 160 & - & 5300 & 27.3 & 7.69 & 18.2 & 30.8 \\
\hline 25 & 500 & 0.95 & - & 28 & 3050 & 1354 & 117 & - & 7552 & 7.69 & 7.69 & 0.00 & 69.2 \\
\hline 25 & 500 & 0.96 & - & 39 & 3050 & 1250 & 117 & - & 6404 & 23.1 & 14.3 & 0.00 & 42.9 \\
\hline 25 & 750 & 0.92 & - & 35 & 950 & 456 & 50.0 & - & 4295 & 34.6 & 2.94 & 3.85 & 32.4 \\
\hline 25 & 750 & 0.95 & - & 52 & 845 & 425 & 50.0 & - & 8314 & 23.3 & 2.78 & 0.00 & 22.2 \\
\hline 25 & 750 & 0.96 & - & 49 & 845 & 425 & 50.0 & - & 4485 & 26.7 & 5.56 & 0.00 & 22.2 \\
\hline 25 & 1000 & 0.92 & - & 82 & 294 & 210 & 14.6 & - & 12406 & 12.7 & 1.64 & 0.00 & 36.1 \\
\hline 25 & 1000 & 0.95 & - & 83 & 286 & 205 & 18.4 & - & 11378 & 10.5 & 1.61 & 1.75 & 29.0 \\
\hline 25 & 1000 & 0.96 & - & 104 & 49.0 & 20.6 & 17.7 & - & 10330 & 6.67 & 1.59 & 0.00 & 47.6 \\
\hline 50 & 100 & 0.92 & - & 11 & 100 & 0.00 & 20 & - & 2805 & 80.0 & 0.00 & 0.00 & 0.00 \\
\hline 50 & 100 & 0.95 & - & 1 & 6280 & 5133 & 40 & - & 2804 & 80.0 & 0.00 & 0.00 & 0.00 \\
\hline 50 & 100 & 0.96 & - & 1 & 6280 & 5133 & 40 & - & 2803 & 80.0 & 0.00 & 0.00 & 0.00 \\
\hline 50 & 250 & 0.92 & - & 1 & 80.0 & 0.00 & 40 & - & 2795 & 40.0 & 0.00 & 0.00 & 0.00 \\
\hline 50 & 250 & 0.95 & - & 1 & 100 & 0.00 & 40 & - & 2796 & 60.0 & 0.00 & 0.00 & 0.00 \\
\hline 50 & 250 & 0.96 & - & 1 & 100 & 0.00 & 40 & - & 2794 & 60.0 & 0.00 & 0.00 & 0.00 \\
\hline 50 & 500 & 0.92 & - & 1 & 6280 & 2143 & 140 & - & 2773 & 100 & 0.00 & 0.00 & 21.4 \\
\hline 50 & 500 & 0.95 & - & 1 & 6280 & 1993 & 160 & - & 2779 & 87.5 & 0.00 & 0.00 & 20.0 \\
\hline 50 & 500 & 0.96 & - & 1 & 6280 & 1993 & 160 & - & 2763 & 87.5 & 0.00 & 0.00 & 20.0 \\
\hline 50 & 750 & 0.92 & - & 8 & 3040 & 913 & 0.00 & - & 2947 & 230 & 6.45 & 0.00 & 3.20 \\
\hline 50 & 750 & 0.95 & - & 10 & 3040 & 773 & 30.0 & - & 2942 & 177 & 0.00 & 0.00 & 22.2 \\
\hline 50 & 750 & 0.96 & - & 8 & 3040 & 749 & 30.0 & - & 2955 & 185 & 0.00 & 0.00 & 18.9 \\
\hline 50 & 1000 & 0.92 & - & 20 & 1470 & 398 & 40.0 & - & 2825 & 125 & 0.00 & 0.00 & 11.1 \\
\hline 50 & 1000 & 0.95 & - & 13 & 1395 & 376 & 42.9 & - & 2880 & 127 & 3.03 & 0.00 & 28.8 \\
\hline 50 & 1000 & 0.96 & - & 14 & 1327 & 355 & 59.1 & - & 2784 & 97.1 & 0.00 & 0.00 & 31.9 \\
\hline
\end{tabular}

Table 2: Computational results, time limit 3000 seconds 
[8] G. Nardini, G. Stea, and A. Virdis. Geofenced broadcasts via centralized scheduling of device-to-device communications in lte-advanced. In S. Balsamo, A. Marin, and E. Vicario, editors, New Frontiers in Quantitative Methods in Informatics, pages 3-17. Springer International Publishing, 2018.

[9] G. Nardini, G. Stea, and A. Virdis. Supporting critical alert services through scheduled device-to-device transmissions in cellular networks. working paper (submitted), 2019.

[10] The ndosolver/fioracle project, https://gitlab.com/frangio68/ndosolver_fioracle_project.

[11] A. Virdis, G. Stea, and G. Nardini. Simulating LTE/LTE-advanced networks with SimuLTE, volume 402 of AISC, pages 83-105. Springer, 2015. 